\title{
CREATINA E TREINAMENTO RESISTIDO: EFEITO NA HIDRATAÇÃO E MASSA CORPORAL MAGRA
}

CREATINE AND RESISTANCE TRAINING: EFFECT ON HYDRATION AND LEAN BODY MASS

CREATINA Y ENTRENAMIENTO RESISTIDO: EFECTO EN LA HIDRATACIÓN Y MASA CORPORAL MAGRA

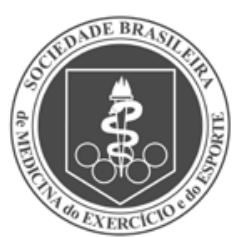

Artigo Original
José Carlos Sales Zanelli (Graduando em Nutrição)

Braian Alves Cordeiro' (Nutricionista)

Bruna Teles Soares Beserra' (Nutricionista)

Erasmo Benício Santos de Moraes Trindade ${ }^{1}$ (Nutricionista)

1. Universidade Federal de Santa Catarina. Florianópolis, SC, Brasil.

\section{Correspondência:}

Erasmo Benício Santos de Moraes Trindade.

Departamento de Nutrição, Centro de Ciências da Saúde, Universidade Federal de Santa Catarina, Campus Universitário, Trindade, 88.040-970, Florianópolis, Brasil. erasmotrindade@gmail.com

\section{RESUMO}

Introdução: a creatina é um recurso ergogênico cuja suplementação tem sido associada ao aumento da hidratação corporal total e ao aumento da massa muscular dos consumidores. Entretanto, estudiosos questionam se o aumento da massa muscular é um ganho real. Objetivo: avaliar o efeito da suplementação de creatina sobre a hidratação e o aumento de massa magra em indivíduos previamente treinados e não treinados, submetidos a um programa de treinamento resistido. Métodos: ensaio clínico não randomizado, constituído por três momentos, M1 - Início da suplementação com 20g/dia de creatina; M2 - 7 dias após iniciada a suplementação e redução da suplementação para 5g/dia; M3 - 28 dias de suplementação. Nos momentos propostos, foram realizadas aferições de peso, estatura e avaliação da composição corporal (massa magra, água corporal total) com a utilização do BYODINAMICS ${ }^{\circledR}$ Modelo 310. Para todos os testes estatísticos, foi adotado o nível de significância de $95 \%$ ( $p<0,05)$. Resultados: participaram desse estudo 14 voluntários adultos do sexo masculino, com idade média de $22,57( \pm 1,45)$ anos, dos quais sete eram treinados e sete não treinados. Após 28 dias de suplementação, no grupo treinado observou-se um aumento significativo no peso, água corporal total, massa magra e hidratação da massa magra, mas nenhum aumento significativo foi observado no grupo não treinado. Em relação ao ângulo de fase, este aumentou no grupo não treinado e reduziu no grupo treinado. Conclusão: a suplementação de creatina associada ao treinamento resistido é mais efetiva na hidratação de indivíduos treinados, como também é suficiente para reduzir a diferença significativa do ângulo de fase intergrupos, sugerindo assim, maior hidratação celular em ambos os grupos. Contudo, esse aumento na hidratação não revelou aumento significativo no tecido muscular.

Palavras-chave: composição corporal, água corporal, fosfocreatina.

\section{ABSTRACT}

Introduction: creatine is an ergogenic aid which supplementation has been associated to increased hydration and increased muscle mass of consumers. However, researchers have questioned whether the increase in muscle mass is a real gain. Objective: to evaluate the effect of creatine supplementation on hydration and increased lean mass in individuals previously trained and untrained, under a resistance training program. Methods: clinical non-randomized study, consisting of three moments, M1 - start of 20g/day creatine supplementation; M2 - 7 days after the beginning of supplementation and reduction to 5g/day; $\mathrm{M3}-28$ days of supplementation. In the proposed moments were made measurements of weight, height and evaluation of body composition (lean mass, total body water) using the BYODINAMICS ${ }^{\circledR}$ Model 310 . For all statistical tests, we used a significance level of 95\% $(p<0.05)$. Results: 14 adult male volunteers with a mean age of 22.57 ( \pm 1.45$)$ years, including seven trained and seven untrained individuals, participated in the study. After 28 days of supplementation, the trained group had a significant increase in weight, total body water, lean body mass and hydration of lean mass, but no significant increase was observed in the untrained group. Regarding the phase angle, it increased in the untrained group and decreased in the trained group. Conclusion: creatine supplementation combined with resistance training is more effective in hydrating trained individuals and it's also sufficient to reduce the difference of the angle phase intergroup, thus suggesting improved cellular hydration in both groups. However, this increase in hydration revealed no significant increase in muscle tissue.

Keywords: body composition, body water, phosphocreatine.

\section{RESUMEN}

Introducción:la creatina es un recurso ergogénico cuya suplementación ha sido asociada al aumento de la hidratación corporal total y al aumento de la masa muscular de los consumidores. Entretanto, estudiosos cuestionan si el aumento de la masa muscular es un aumento real. Objetivo: evaluar el efecto de la suplementación de creatina sobre la hidratación y el aumento de masa magra en individuos previamente entrenados y no entrenados, sometidos a un programa de entrenamiento resistido. Métodos: ensayo clínico no aleatorio, constituido por tres momentos, M1 - Inicio de la suplementación con 20g/día de creatina; M2 - 7 días después de iniciada la suplementación y reducción de la suplementación para 5g/día; M3 - 28 días de suplementación. En los momentos propuestos, fueron realizadas mediciones de peso, estatura y evaluación de la composición corporal 
(masa magra, agua corporal total) con el uso de BYODINAMICS ${ }^{\oplus}$ Modelo 310. Para todos los tests estadísticos, fue adoptado el nivel de significancia de 95\% ( $p<0,05)$. Resultados: participaron en este estudio 14 voluntarios adultos del sexo masculino, con edad promedio de 22,57( $\pm 1,45)$ años, de los que siete eran entrenados y siete no entrenados. Después de 28 días de suplementación, en el grupo entrenado se observó un aumento significativo en el peso, agua corporal total, masa magra e hidratación de la masa magra, pero ningún aumento significativo fue observado en el grupo no entrenado. En relación al ángulo de fase, aumentó en el grupo no entrenado y se redujo en el grupo entrenado. Conclusión: la suplementación de creatina asociada al entrenamiento resistido es más efectiva en la hidratación de individuos entrenados, como también es suficiente para reducir la diferencia significativa del ángulo de fase intergrupos, sugiriendo asi mayor hidratación celular en ambos grupos. Sin embargo, ese aumento en la hidratación no reveló aumento significativo en el tejido muscular.

Palabras clave: composición corporal, agua corporal, fosfocreatina.

\section{INTRODUÇÃO}

A creatina é um derivado de aminoácidos de uso frequente entre atletas e praticantes de atividade física, em especial aqueles que praticam modalidades de alta intensidade e curta duração ${ }^{1-3}$. O principal objetivo desses atletas é se beneficiar do efeito ergogênico da creatina, com o possível ganho de massa muscular e a melhoria no desempenho físico ${ }^{4,5}$. Em humanos, a creatina é armazenada principalmente na musculatura esquelética (95\%) e, em menor quantidade, no cérebro, fígado, rins e testículos ${ }^{6}$.

A utilização da creatina em forma de suplemento alimentar vem sendo estudada em diversos campos da saúde e com públicos diferenciados como idosos, enfermos, atletas e praticantes de atividade física. Dentre os resultados positivos já encontrados com a utilização da creatina, estão a melhora entre portadores de miopatias inflamatórias, distrofias musculares, síndromes de deficiência em creatina, condicionamento pré e pós isquêmico, déficit cognitivo e também a melhora no desempenho de praticantes de atividade física e atletas ${ }^{3,7}$.

Em relação aos praticantes de atividade física, é oportuno definir o Treinamento Resistido (TR), que é uma educação para suportar exercícios sistemáticos submetidos a um esforço contrário e é um método essencial nos programas de treinamento para indivíduos com os mais diversos objetivos e níveis de aptidão física ${ }^{8,9}$. OTR é considerado seguro para indivíduos saudáveis, para atletas e para portadores de doenças crônicas ${ }^{9}$. Uma vez considerada a capacidade de adaptação pessoal, esse treinamento pode auxiliar na potência, força, redução do risco de lesões, resistência física e desempenho ${ }^{10}$.

Quando associado à suplementação de creatina, o TR tem mostrado maior beneficio para o aumento de massa corporal, força máxima e capacidade de elevação de peso em relação ao TR sem a suplementação. Embora sejam esses os dados mais frequentemente observados, as respostas são variáveis em ganho e intensidade de ganho ${ }^{4}$.

O aumento de massa magra associada à suplementação de creatina tem sido alvo de divergência na literatura quanto ao fator responsável por esse ganho. $\mathrm{O}$ aumento de massa magra e peso corporal foram justificados em muitos estudos apenas pela retenção hídrica decorrentes do uso do suplemento. Estes indicam que a suplementação de creatina aumenta o peso corporal na primeira semana de carregamento de creatina devido à sua característica osmótica1,6,11-13. Contudo, estudiosos questionam se as mudanças nos conteúdos intracelulares de água podem influenciar a tradução de proteínas contráteis e justificar parte do real ganho de massa magra ${ }^{5,14,15}$.

Diante do exposto, o presente estudo tem como objetivo avaliar o efeito da suplementação de creatina sobre a hidratação e o aumento de massa magra em homens treinados e não treinados submetidos ao treinamento resistido.

\section{MÉTODOS}

Ensaio clínico não randomizado realizado no município de Florianópolis, Estado de Santa Catarina - Brasil. Foram avaliados indivíduos frequentadores de uma academia de musculação. A pesquisa foi aprovada pelo Comitê de Ética e Pesquisa em Seres Humanos da Universidade Federal de Santa Catarina (CEPSH/UFSC), aprovado sob o número 412.502/2013. Não obstante, esta pesquisa foi elaborada em conformidade com a resolução do Conselho Nacional de Saúde n466/12. Todos os indivíduos envolvidos neste estudo foram informados e assinaram o Termo de Consentimento Livre e Esclarecido para anuência da execução do mesmo. No presente estudo não há conflitos de interesse.

Foram incluídos no estudo indivíduos que realizavam programa de treinamento resistido há pelo menos sete dias, com frequência mínima de três vezes por semana. Foram excluídos do estudo os voluntários que declararam ter ingerido, nos últimos 60 dias, medicamentos anti-inflamatórios esteroidais; esteroides anabolizantes; qualquer substância classificada como diurética além da cafeína encontrada na dieta habitual; ou qualquer outro suplemento que contenha creatina em sua composição; ser portador de marca-passo; e/ou ser portador de doença renal.

O suplemento utilizado foi a creatina monohidratada com as seguintes características nutricionais: Porção - 5g (01 envelope), Valor energético - $20 \mathrm{kcal}=84 \mathrm{~kJ}$, Proteínas $-5 \mathrm{~g}$, Creatina monohidratada 5000 mg. O suplemento era acondicionado em envelopes idênticos, de mesmo lote de produção, em doses de cinco gramas cada. A creatina foi doada por farmácia de manipulação de Florianópolis SC, Brasil.

O estudo foi constituído por três momentos (M): M1 - início da suplementação com 20g/dia de creatina; M2 - 7 dias após a suplementação e início da suplementação com 5g/dia; M3 - 28 dias após a suplementação.

Nesse período foram realizadas três baterias para aferição das medidas nos momentos propostos. Em M1 e M2, os participantes foram orientados a manter a restrição de anti-inflamatórios esteroidais e não-esteroidais, esteroides anabolizantes, suplementos alimentares que contivessem creatina em sua composição e fármacos diuréticos ou qualquer substância classificada como diurética que não fosse a cafeína usual da dieta.

A suplementação foi administrada em dois momentos. No M1, com duração de sete dias, os participantes seguiram um regime de 20 gramas (g) de creatina monohidratada ao dia, administrados em doses de cinco gramas a cada quatro horas. No M2 e ao longo das três semanas seguintes, a dose de creatina foi reduzida para $5 \mathrm{~g} / \mathrm{dia}$, administrada em dose única no horário imediatamente pós-treino. Nos dias sem treino, a orientação foi de ingerir o suplemento no horário 
correspondente ao pós-treino. A creatina foi ingerida após diluição do envelope em $250 \mathrm{ml}$ ou um copo americano de água.

Foi aplicado um questionário individual para avaliar a elegibilidade dos voluntários, conforme os critérios de inclusão e exclusão acima referidos. Os indivíduos selecionados foram distribuídos em dois grupos de sete voluntários, sendo um de indivíduos treinados (GT) e outro de não treinados (GNT). Todos os indivíduos selecionados concordaram em interromper o treinamento que vinham realizando na academia para participar apenas do programa oferecido pelo estudo, que consistiu na associação de suplementação por creatina e um protocolo de treinamento resistido controlado durante 28 dias.

O protocolo de treinamento resistido foi elaborado por um profissional de educação física e caracterizado como treinamento neuromuscular (musculação) com objetivo de hipertrofia. Foram programadas três rotinas de treino, para diferentes grupos musculares: (a) peitorais, tríceps e abdômen; (b) dorsais e bíceps; e (c) deltoides, quadríceps, femorais, gastrocnêmio e sóleo. $O$ volume médio foi de quatro exercícios por grupo muscular, cada um deles com 12 séries de 10 a 12 repetições máximas até a falha concêntrica. 0 intervalo entre as séries foi de 40-60 segundos e a cadência utilizada foi de 1 segundo para a fase concêntrica e 3 segundos para a fase excêntrica. Os voluntários foram instruídos a realizar o treinamento pelo menos três vezes por semana e em horário fixo. Atividades aeróbicas realizadas fora da academia, de baixo impacto e esporádicas, não foram controladas.

No momento 1 foi aferida a estatura dos participantes. Em todos os momentos do estudo foi aferido o peso corporal e realizada a bioimpedância para determinação do peso de massa magra, quantidade de água corporal total, porcentagem de hidratação da massa magra e cálculo do ângulo de fase. Para realização desses procedimentos foi utilizado uma balança mecânica com estadiômetro acoplado, modelo $110 \mathrm{CH}$ da marca Welmy ${ }^{\circledR}$, e o aparelho BYODINAMICS ${ }^{\circledR}$ Modelo 310 (TBW, Rua Edward Joseph, 122 - cj. 42 - Morumbi, São Paulo/SP).

Para a realização da bioimpedância, os participantes foram orientados a ter uma noite de repouso na véspera; não realizar nenhum tipo de atividade física 12 horas antes; não consumir chocolates, cafés, chás ou bebidas energéticas 24 horas antes; e comparecer em jejum de quatro horas de alimentos e de bebidas.

\section{Análise estatística}

Os dados foram organizados e registrados em banco de dados no programa Microsoft Office Exce ${ }^{\circledR} 2010$ e analisados no Software STATA ${ }^{\circledR} 11.0$ versão para Windows. Para avaliar a simetria das variáveis foi considerado o coeficiente de variação e o teste de Shapiro-wilk (valores de $\mathrm{p}<0,05$ são considerados variáveis assimétricas). Os dados foram apresentados em média e desvio-padrão para variáveis simétricas. Foi utilizado teste $t$ de Student ou de Mann Whitney para comparar desfechos contínuos intergrupos em distribuição simétrica com homogeneidade das variâncias e distribuiçãosimétrica com heterogeneidade das variâncias, respectivamente. teste T pareado foi aplicado para detectar diferenças intragrupo nos momentos quando variável de desfecho apresentou-se simétrica.

Este estudo teve o objetivo de investigar o efeito da suplementação de creatina sobre as medidas da composição corporal em indivíduos treinados e não-treinados.

\section{RESULTADOS}

Participaram do estudo 14 voluntários adultos do sexo masculino,

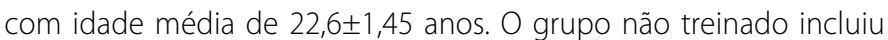
voluntários com mediana do tempo de treinamento resistido igual a seis semanas sendo que 50\% tem treinamento entre 2 e 16 semanas e o grupo treinado incluiu aqueles com tempo médio de treinamento resistido de 5,1 1 1,46 anos.

A composição corporal dos participantes do estudo apresentou o seguinte comportamento nos diferentes momentos: em M1 e M2 ocorreu o maior aumento no peso, massa magra, água total e hidratação da massa magra, entretanto o ângulo de fase obteve menor ganho; em M2 e M3 houve aumento não significativo na hidratação da massa magra e ângulo de fase e para os demais parâmetros houve redução nos valores, também não significativo; em M1 e M3 observou-se aumento significativo $(p<0,05)$ na média de peso corporal, massa magra, água total e hidratação da massa magra (tabela 1).

A comparação intergrupos mostra aumento, da pré-suplementação ao fim da suplementação, para os dois grupos em todas as medidas da composição corporal, sem diferença significativa entre eles. A aferição realizada antes da suplementação, em M1, mostrou valor significativamente maior $(p<0,05)$ para o ângulo de fase no $G T$, que não foi mais observada após o início da suplementação. As demais medidas iniciais também foram superiores no GT, mas com diferença não significativa. Iniciado o programa, a aferição em M2 mostrou aumento $(p<0,05)$ da porcentagem de hidratação da massa magra no GT em relação ao GNT. O valor de ângulo de fase aumentou para o GNT e reduziu para GT. Nos demais parâmetros também houve aumento para os dois grupos, com diferença não significativa. Quando comparadas as medidas no M2 e M3, é observado aumento de peso no GNT, enquanto a mesma foi reduzida no GT. Por outro lado, houve aumento nas medidas de hidratação da massa magra no GT e redução dessas medidas no GNT. Ambos os grupos apresentaram ganhos nas medidas de ângulo de fase e perdas na massa magra e água corporal total (tabela 2).

Para o grupo treinado, houve aumento de peso (5,5\%), água total $(4,7 \%)$ e da porcentagem de hidratação da massa magra $(0,4 \%)(p<0,005)$ ao final do programa, sendo que para o peso o ganho já foi significativo ao final dos primeiros sete dias. As medidas de massa magra aumentaram $(5,9 \%)$ nos primeiros sete dias, seguido de perda nas três semanas seguintes (1,9\%), ambas variações significativas. Essa variação resultou em um ganho final não significativo entre M1 e M3. Para o ângulo de fase houve diminuição entre $\mathrm{M} 1$ e $\mathrm{M} 2$ (0,4\%), com posterior aumento em M3 $(1,6 \%)$, sendo todas as variações não significativas (tabela 3$)$.

Para o grupo não-treinado houve aumento $(p<0,05)$ nos valores de massa magra (4,5\%), água total $(5,3 \%)$ e ângulo de fase $(3,4 \%)$ durante os primeiros sete dias. Ao longo do período de manutenção houve perda nesses parâmetros, resultando em um ganho não significativo ao final do programa. A comparação entre as aferições realizadas em M1 e M3 mostra que houve ganho em todos os parâmetros, mas nenhum deles significativo (tabela 4).

Tabela 1. Parâmetros de composição corporal dos participantes do estudo em $M 1, M 2$ e M3.

\begin{tabular}{|c|c|c|c|c|c|c|c|c|c|}
\hline Variáveis & $\begin{array}{c}\text { M1 } \\
\text { Média } \\
\pm \mathrm{DP} \\
\end{array}$ & $\begin{array}{c}\text { M2 } \\
\text { Média } \\
\pm \text { DP }\end{array}$ & $p$ & \begin{tabular}{|c|} 
M2 \\
Média \\
$\pm \mathrm{DP}$ \\
\end{tabular} & $\begin{array}{c}\text { M3 } \\
\text { Média } \\
\pm \text { DP }\end{array}$ & $p$ & \begin{tabular}{|c|} 
M1 \\
Média \\
$\pm \mathrm{DP}$ \\
\end{tabular} & $\begin{array}{c}\text { M3 } \\
\text { Média } \\
\pm \text { DP } \\
\end{array}$ & $p$ \\
\hline Peso ( & $\begin{array}{r}77,89 \\
\pm 11,69 \\
\end{array}$ & $\begin{array}{r}81,60 \\
\pm 10,91\end{array}$ & t & $\begin{array}{c}81,60 \\
\pm 10,91 \\
\end{array}$ & $\begin{array}{l}81,20 \\
\pm 9,61\end{array}$ & $03^{*}$ & $\begin{array}{r}77,89 \\
\pm 11,69 \\
\end{array}$ & $\begin{array}{l}81,20 \\
\pm 9,61\end{array}$ & $0,008^{*}$ \\
\hline$M$ & $\begin{array}{l}66,98 \\
\pm 7,23\end{array}$ & $\begin{array}{l}70,27 \\
\pm 6,53\end{array}$ & $03^{*}+$ & $\begin{array}{l}70,27 \\
\pm 6,53\end{array}$ & $\begin{array}{r}69,91 \\
\pm 6,91\end{array}$ & $304^{*}$ & $\begin{array}{l}66,98 \\
\pm 7,23\end{array}$ & $\begin{array}{l}69,91 \\
\pm 6,91\end{array}$ & $0,014^{*}$ \\
\hline $\begin{array}{c}\text { Água total } \\
(L)\end{array}$ & $\begin{array}{l}47,46 \\
\pm 5,72\end{array}$ & $\begin{array}{l}50,27 \\
\pm 5,46\end{array}$ & $0,005^{*}+$ & $\begin{array}{l}50,27 \\
\pm 5,46\end{array}$ & $\begin{array}{l}50,15 \\
\pm 6,17\end{array}$ & $0,274^{*}$ & $\begin{array}{l}47,46 \\
\pm 5,72\end{array}$ & $\begin{array}{l}50,15 \\
\pm 6,17\end{array}$ & 0,017 \\
\hline $\begin{array}{c}\text { Hidratação } \\
\text { da MCM } \\
(\%)\end{array}$ & $\begin{array}{l}70,80 \\
\pm 1,31\end{array}$ & $\begin{array}{l}71,43 \\
\pm 1,44\end{array}$ & $*^{*}$ & $\begin{array}{l}71,43 \\
\pm 1,44\end{array}$ & $\begin{array}{l}71,60 \\
\pm 2,04\end{array}$ & $0,280^{*}$ & $\begin{array}{r}70,80 \\
\pm 1,31\end{array}$ & $\begin{array}{l}71,60 \\
\pm 2,04\end{array}$ & 0,04 \\
\hline $\begin{array}{c}\text { Ângulo de } \\
\text { fase }\left({ }^{\circ}\right)\end{array}$ & $\begin{array}{c}7,83 \\
\pm 0,91\end{array}$ & $\begin{array}{c}7,91 \\
\pm 0,83\end{array}$ & $0,344^{*}$ & $\begin{array}{c}7,91 \\
\pm 0,83\end{array}$ & $\begin{array}{c}8,15 \\
\pm 0,81\end{array}$ & $0,381^{*}$ & $\begin{array}{c}7,83 \\
\pm 0,91\end{array}$ & $\begin{array}{c}8,15 \\
\pm 0,81\end{array}$ & 0,089 \\
\hline
\end{tabular}


Tabela 2. Comparação dos parâmetros da composição corporal entre o grupo treinado e o grupo não treinadonos três momentos do estudo.

\begin{tabular}{|c|c|c|c|c|c|c|}
\hline Variáveis & $\begin{array}{c}\text { M1 } \\
\text { Média } \\
\text { 土 DP }\end{array}$ & $p$ & $\begin{array}{c}\text { M2 } \\
\text { Média } \pm \mathrm{DP}\end{array}$ & $p$ & $\begin{array}{c}\text { M3 } \\
\text { Média } \\
\text { 土DP }\end{array}$ & $p$ \\
\hline \multicolumn{7}{|l|}{ Peso (kg) } \\
\hline GNT & $75,84 \pm 15,89$ & \multirow{2}{*}{$0,179^{* *}$} & $79,83 \pm 15,29$ & \multirow{2}{*}{$0,584^{*}$} & $80,02 \pm 13,01$ & \multirow{2}{*}{$0,699 *$} \\
\hline GT & $79,94 \pm 5,80$ & & $83,72 \pm 5,66$ & & $82,21 \pm 6,40$ & \\
\hline \multicolumn{7}{|l|}{ MCM (kg) } \\
\hline GNT & $65,00 \pm 8,58$ & & $67,95 \pm 7,29$ & \multirow{2}{*}{$0,212^{*}$} & $67,82 \pm 6,75$ & \multirow{2}{*}{$0,334^{*}$} \\
\hline GT & $68,96 \pm 5,54$ & & $73,06 \pm 4,72$ & & $71,70 \pm 7,03$ & \\
\hline \multicolumn{7}{|l|}{$\begin{array}{c}\text { Água } \\
\text { corporal } \\
\text { total (L) }\end{array}$} \\
\hline GNT & $45,61 \pm 6,17$ & \multirow{2}{*}{$0,240^{*}$} & $48,02 \pm 5,52$ & \multirow{2}{*}{$0,140^{*}$} & $47,77 \pm 5,03$ & \multirow{2}{*}{$0,209^{*}$} \\
\hline GT & $49,31 \pm 4,98$ & & $52,98 \pm 4,45$ & & $52,20 \pm 6,67$ & \\
\hline \multicolumn{7}{|l|}{$\begin{array}{l}\text { Hidratação } \\
\text { da MCM(\%) }\end{array}$} \\
\hline GNT & $70,14 \pm 0,54$ & \multirow{2}{*}{$0,141^{* *}$} & $70,60 \pm 0,64$ & \multirow{2}{*}{$0,026^{*}+$} & $70,40 \pm 0,56$ & \multirow{2}{*}{$0,115^{* *}$} \\
\hline GT & $71,46 \pm 1,56$ & & $72,42 \pm 1,55$ & & $72,63 \pm 2,32$ & \\
\hline \multicolumn{7}{|l|}{$\begin{array}{c}\text { Ângulo de } \\
\text { fase }\left({ }^{\circ}\right)\end{array}$} \\
\hline GNT & $7,30 \pm 0,66$ & \multirow[t]{2}{*}{$0,020^{*} \dagger$} & $7,55 \pm 0,81$ & \multirow[t]{2}{*}{$0,122^{*}$} & $7,77 \pm 0,62$ & \multirow[t]{2}{*}{$0,124^{*}$} \\
\hline GT & $8,37 \pm 0,83$ & & $8,34 \pm 0,70$ & & $8,47 \pm 0,85$ & \\
\hline
\end{tabular}

MCM: Massa corporal magra; M1: momento 1; M2: momento 2; M3: momento 3*Teste T; **Teste Mann-Whitney; $+\mathrm{p}<0,05$

Tabela 3. Parâmetros da composição corporal do grupo treinado nos diferentes momentos do estudo.

\begin{tabular}{|c|c|c|c|c|c|c|c|c|c|}
\hline Variáveis & $\begin{array}{c}\text { M1 } \\
\text { Média } \\
\pm \mathrm{DP}\end{array}$ & $\begin{array}{c}\text { M2 } \\
\text { Média } \\
\pm \text { DP }\end{array}$ & $p$ & $\begin{array}{c}\text { M2 } \\
\text { Média } \\
\pm \text { DP }\end{array}$ & $\begin{array}{c}\text { M3 } \\
\text { Média } \\
\pm \mathrm{DP}\end{array}$ & $p$ & $\begin{array}{c}\text { M1 } \\
\text { Média } \\
\pm \mathrm{DP}\end{array}$ & $\begin{array}{c}\text { M3 } \\
\text { Média } \\
\pm \mathrm{DP}\end{array}$ & $p$ \\
\hline Peso (kg) & \begin{tabular}{|l|}
79,94 \\
$\pm 5,80$ \\
\end{tabular} & $\begin{array}{r}83,72 \\
\pm 5,66 \\
\end{array}$ & $0,022^{*}+$ & $\begin{array}{l}83,72 \\
\pm 5,66 \\
\end{array}$ & $\begin{array}{l}82,21 \\
\pm 6,40 \\
\end{array}$ & $0,059^{*}$ & \begin{tabular}{|l|}
79,94 \\
$\pm 5,80$ \\
\end{tabular} & \begin{tabular}{|l|}
82,21 \\
$\pm 6,40$ \\
\end{tabular} & $0,003^{*} \dagger$ \\
\hline MCM (kg) & \begin{tabular}{|l|}
68,96 \\
$\pm 5,54$ \\
\end{tabular} & $\begin{array}{r}73,06 \\
\pm 4,72 \\
\end{array}$ & $0,017^{*}+$ & $\begin{array}{r}73,06 \\
\pm 4,72 \\
\end{array}$ & $\begin{array}{r}71,70 \\
\pm 7,03 \\
\end{array}$ & $0,017^{*}+$ & \begin{tabular}{|l|}
68,96 \\
$\pm 5,54$ \\
\end{tabular} & \begin{tabular}{|l|}
71,70 \\
$\pm 7,03$ \\
\end{tabular} & $0,184^{*}$ \\
\hline $\begin{array}{c}\text { Água total } \\
\text { (L) }\end{array}$ & \begin{tabular}{|l|}
49,31 \\
$\pm 4,98$ \\
\end{tabular} & $\begin{array}{l}52,98 \\
\pm 4,45 \\
\end{array}$ & $0,179 *$ & $\begin{array}{l}52,98 \\
\pm 4,45 \\
\end{array}$ & $\begin{array}{l}52,20 \\
\pm 6,67 \\
\end{array}$ & $0,380^{*}$ & \begin{tabular}{|l|}
49,31 \\
$\pm 4,98$ \\
\end{tabular} & \begin{tabular}{|l|}
52,20 \\
$\pm 6,67$ \\
\end{tabular} & $0,042^{*} \dagger$ \\
\hline $\begin{array}{r}\text { Hidratação } \\
\text { da MCM (\%) }\end{array}$ & $\begin{array}{l}71,46 \\
\pm 1,56\end{array}$ & $\begin{array}{r}72,42 \\
\pm 1,55\end{array}$ & $0,179 *$ & $\begin{array}{l}72,42 \\
\pm 1,55\end{array}$ & $\begin{array}{l}72,63 \\
\pm 2,32\end{array}$ & $0,092^{*}$ & $\begin{array}{l}71,46 \\
\pm 1,56\end{array}$ & \begin{tabular}{|l|}
72,63 \\
$\pm 2,32$ \\
\end{tabular} & $0,043^{*} \dagger$ \\
\hline $\begin{array}{c}\text { Ângulo de } \\
\text { fase (o) }\end{array}$ & \begin{tabular}{|c|}
8,37 \\
$\pm 0,83$ \\
\end{tabular} & $\begin{array}{c}8,34 \\
\pm 0,70 \\
\end{array}$ & $0,477^{*}$ & $\begin{array}{c}8,34 \\
\pm 0,70 \\
\end{array}$ & $\begin{array}{c}8,47 \\
\pm 0,85 \\
\end{array}$ & $0,898^{*}$ & \begin{tabular}{|c|}
8,37 \\
$\pm 0,83$ \\
\end{tabular} & \begin{tabular}{|c|}
8,47 \\
$\pm 0,85$ \\
\end{tabular} & $0,629^{*}$ \\
\hline
\end{tabular}

Tabela 4. Parâmetros da composição corporal do grupo não treinado nos diferentes momentos do estudo.

\begin{tabular}{|c|c|c|c|c|c|c|c|c|c|}
\hline Variáveis & $\begin{array}{c}\text { M1 } \\
\text { Média } \\
\pm \mathrm{DP}\end{array}$ & $\begin{array}{c}\text { M2 } \\
\text { Média } \\
\pm \text { DP }\end{array}$ & $p$ & $\begin{array}{c}\text { M2 } \\
\text { Média } \\
\pm \mathrm{DP}\end{array}$ & $\begin{array}{c}\text { M3 } \\
\text { Média } \\
\pm \mathrm{DP}\end{array}$ & $p$ & $\begin{array}{c}\text { M1 } \\
\text { Média } \\
\pm \text { DP }\end{array}$ & $\begin{array}{c}\text { M3 } \\
\text { Média } \\
\pm \text { DP }\end{array}$ & $p$ \\
\hline Peso (kg) & \begin{tabular}{|c|}
75,84 \\
$\pm 15,89$ \\
\end{tabular} & \begin{tabular}{|r|}
79,83 \\
$\pm 15,29$ \\
\end{tabular} & $0,116^{*}$ & \begin{tabular}{|c|}
79,83 \\
$\pm 15,29$ \\
\end{tabular} & \begin{tabular}{|c|}
80,02 \\
$\pm 13,01$ \\
\end{tabular} & $0,833^{*}$ & $\begin{array}{r}75,84 \\
\pm 15,89 \\
\end{array}$ & \begin{tabular}{|c|}
80,02 \\
$\pm 13,01$ \\
\end{tabular} & $0,600 *$ \\
\hline MCM (kg) & \begin{tabular}{|l|}
65,00 \\
$\pm 8,58$ \\
\end{tabular} & $\begin{array}{l}67,95 \\
\pm 7,29\end{array}$ & $0,035^{*}+$ & $\begin{array}{l}67,95 \\
\pm 7,29\end{array}$ & $\begin{array}{r}67,82 \\
\pm 6,75\end{array}$ & $0,345^{*}$ & $\begin{array}{r}65,00 \\
\pm 8,58\end{array}$ & \begin{tabular}{|l|}
67,82 \\
$\pm 6,75$ \\
\end{tabular} & $0,528^{*}$ \\
\hline $\begin{array}{c}\text { Água total } \\
\text { (L) }\end{array}$ & $\begin{array}{l}45,61 \\
\pm 6,17 \\
\end{array}$ & $\begin{array}{l}48,02 \\
\pm 5,52 \\
\end{array}$ & $0,046^{*}+$ & $\begin{array}{l}48,02 \\
\pm 5,52 \\
\end{array}$ & \begin{tabular}{|l|}
47,77 \\
$\pm 5,03$
\end{tabular} & $0,399 *$ & \begin{tabular}{|}
45,61 \\
$\pm 6,17$ \\
\end{tabular} & \begin{tabular}{|l|}
47,77 \\
$\pm 5,03$ \\
\end{tabular} & $0,600 *$ \\
\hline $\begin{array}{l}\text { Hidratação } \\
\text { da MCM (\%) }\end{array}$ & \begin{tabular}{|l|}
70,14 \\
$\pm 0,54$ \\
\end{tabular} & $\begin{array}{l}70,60 \\
\pm 0,64\end{array}$ & $0,092^{*}$ & $\begin{array}{r}70,60 \\
\pm 0,64\end{array}$ & $\begin{array}{l}70,40 \\
\pm 0,56 \\
\end{array}$ & $0,527^{*}$ & \begin{tabular}{|l|}
70,14 \\
$\pm 0,54$ \\
\end{tabular} & \begin{tabular}{|l|}
70,40 \\
$\pm 0,56$ \\
\end{tabular} & $0,673^{*}$ \\
\hline $\begin{array}{c}\text { Ângulo de } \\
\text { fase (o) }\end{array}$ & \begin{tabular}{|c|}
7,30 \\
$\pm 0,66$ \\
\end{tabular} & $\begin{array}{r}7,55 \\
\pm 0,81\end{array}$ & $0,034^{*} \dagger$ & $\begin{array}{c}7,55 \\
\pm 0,81\end{array}$ & $\begin{array}{c}7,77 \\
\pm 0,62\end{array}$ & $0,292^{*}$ & \begin{tabular}{|c|}
7,30 \\
$\pm 0,66$ \\
\end{tabular} & \begin{tabular}{|c|}
7,77 \\
$\pm 0,62$ \\
\end{tabular} & $0,116^{*}$ \\
\hline
\end{tabular}

\section{DISCUSSÃO}

Os resultados mostraram que houve efetivo aumento dessas medidas nos participantes do estudo, uma vez que os dados apresentaram variação significativa durante os primeiros sete dias de programa. Ainda, quando sujeitos à fase de manutenção, houve um decréscimo não significativo nas medidas.

Os dados encontrados estão de acordo com outros estudos ${ }^{1,4,11-13,16-19}$, nos quais utilizaram protocolo semelhante, usando 20 a $25 \mathrm{~g} /$ dia de creatina durante 5 a 7 dias, e demonstraram aumento de peso já no término da fase de carregamento de creatina. No estudo de Powers et al. ${ }^{1}$, homens e mulheres treinados receberam 25g/dia por sete dias em fase de carregamento com creatina monohidratada, em que os participantes tiveram aumento no peso $(75,54 \pm 17,67 \mathrm{~kg}$ e 76,29 $\pm 18,04 \mathrm{~kg})$, água corporal total $(41,98 \pm 11,78 \mathrm{~L}$ e 43,35 $\pm 12,19 \mathrm{~L})$ e após a fase de manutenção ( $5 \mathrm{~g} /$ dia por 21 dias), mantiveram de forma semeIhante os parâmetros aumentados de peso $(76,86 \pm 18,07 \mathrm{~kg})$, água total $(44,02 \pm 12,37 \mathrm{~L})$ quando comparado ao momento pré-suplementação. Já no estudo de Easton et al. ${ }^{11}$, homens treinados de endurance também apresentaram aumento do peso corporal e da água total após receberem 23g/dia de creatina monohidratada por sete dias, em que o peso e a água total aumentaram em 0,9kg e 0,7L. Ambos os estudos utilizaram bioimpedância elétrica para avaliar a água corporal total (Xitron Technologies Inc, San Diego, CA e Quadscan 4000, BodystatLtd., Isleof Man). Os estudos concluíram que o balanço hidroeletrolítico não é afetado com a suplementação de creatina, pois o aumento de água corporal seria proporcional para água intra e extracelular.

A diferença de água corporal total encontrada entre $\mathrm{M} 1$ e M3, bem como M1 e M2 foram semelhantes à diferença de peso corporal e massa corporal magra. Apesar da diferença significativa em vários parâmetros, o aumento da água corporal parece ter sido o principal efeito causado pela suplementação de creatina e o responsável pelo aumento dos demais parâmetros. Esse efeito é justificado em estudos devido à creatina exercer efeito osmótico e com isso, ocorre o movimento de água para dentro da célula, a retenção de água e o ganho de peso 1,13,16. No estudo de Bemben et al.13 a suplementação de creatina resultou no aumento de 3,3 litros $(9,0 \%)$ a mais na média de água corporal total do grupo suplementado, demonstrando o efeito no aumento da hidratação da suplementação de creatina.

Contudo, outros autores mostram que o aumento dos estoques de creatina fosfato pode exercer efeito sobre a transcrição de fatores miogênicos nas vias hipertróficas PI3K-AKT/PKB-mTOR e propiciar aumento de síntese proteica e possível tecido muscular, consequentemente ${ }^{5,20,21}$. Dessa forma, um aumento de massa magra independente de hiper-hidratação poderia ser notado.

O principal achado nesse estudo foi observar que, ao comparar participantes do GNT e GT, os parâmetros de peso e MCM não tiveram diferença significativa na pré-suplementação (M1), final da fase de carregamento (M2) e após 28 dias de suplementação (M3). Esses resultados sugerem que o nível de treinamento dos praticantes de musculação não exerce influência nesses parâmetros para a suplementação de creatina. Ainda comparando os resultados de GT e GNT, foi constatado maior aumento da porcentagem de hidratação da massa magra entre os indivíduos treinados após a fase de carregamento, mesmo sem ter aumento significativo da água corporal total. Além disso, o ângulo de fase no momento pré-suplementação dos indivíduos treinados foi maior que o dos indivíduos não-treinados, o que pode ter relação com o grau de hidratação celular entre os grupos.

O ângulo de fase dos indivíduos do GNT apresentou média mais baixa $(7,30 \pm 0,66)$ que os indivíduos do $\mathrm{GT}(8,37 \pm 0,83)$ no momento pré-suplementação com diferença significativa entre os grupos $(p=0,020)$. 
De acordo com Selberg e Selberg ${ }^{22}$, o ângulo de fase (AF) é uma relação entre as grandezas resistência e reatância e é determinado pela celularidade tecidual, hidratação tecidual e potencial de membrana, em que ângulos de fase baixos sugerem morte celular ou decréscimo na integridade celular, enquanto ângulos de fase elevados sugerem grandes quantidades de membranas celulares intactas. Barbosa-Silva et al.23, estudaram 1967 indivíduos americanos saudáveis, com idade entre 18 e 94 anos, cujo AF foi significativamente maior nos homens do que nas mulheres $\left(7,48 \pm 1,10^{\circ}\right.$ e $6,53 \pm 1,10^{\circ}$, respectivamente). Esse valor diminuiu com a idade para 35 participantes, com redução de $7,90^{\circ}$ (grupo mais novo) para 6,19० (grupo mais velho) nos homens e de 7,040 (grupo mais novo) para 5,640 (grupo mais velho) nas mulheres.

Analisando M2 e M3 deste estudo, percebe-se que não houve mais diferença significativa ( $p=0,122$ e $p=0,124)$. Considerando a relação do ângulo de fase com a hidratação celular, esses resultados sugerem que o grupo GNT iniciou o estudo em um nível de hidratação celular inferior ao do grupo GT, e que após suplementação de creatina, o ângulo de fase de GNT aumentou para uma faixa de hidratação mais próxima de GT.

Com relação à porcentagem de hidratação da massa magra, GT apresentou $71,46 \pm 1,56$ enquanto GNT apresentou $70,14 \pm 0,54$ no momento pré-suplementação, sem diferença significativa. Já no momento M2 (final da fase de carregamento), GT aumentou em maior proporção seus valores $(72,42 \pm 1,55)$ em relação à GNT $(70,60 \pm 0,64)$, o que resultou em um $p=0,026$. Contudo, no momento M3 (final da suplementação), não houve diferença significativa ( $p=0115)$ entre $G T(72,63 \pm 2,62)$ e GNT $(70,40 \pm 0,56)$. Esses resultados levantam a hipótese de que indivíduos treinados podem aumentar a hidratação da massa magra em tempo inferior aos indivíduos não treinados.

\section{CONCLUSÃO}

A suplementação de creatina associada ao TR por 28 dias trouxe ganho significativo para peso, ACT e hidratação da massa magra para o GT, mas nenhum aumento significativo para o GNT. Contudo, todos os parâmetros de ambos os grupos foram aumentados ao final da suplementação. O ângulo de fase aumentou mais no GNT que no GT percentualmente, porém o aumento da hidratação da massa magra foi maior no GT do que em GNT. O nível de treinamento dos indivíduos talvez exerça influência sobre a intensidade do efeito da creatina no aumento da hidratação celular e muscular. Contudo, esse efeito não mostrou ligação com o aumento de tecido muscular em nenhum dos grupos, visto que o aumento de massa magra correspondeu aproximadamente ao aumento de água corporal total em ambos os grupos.

\section{AGRADECIMENTOS}

À doação do suplemento pela farmácia de manipulação Vital Farm.

Todos os autores declararam não haver qualquer potencial conflito de interesses referente a este artigo.

\section{REFERÊNCIAS}

1. Powers ME, Arnold BL, Weltman AL, Perrin DH, Mistry D, Kahler DM, et al. Creatine supplementation increases total body water without altering fluid distribution. J Athl Train. 2003;38(1):44-50.

2. Carvalho APPF, Molina GE, Fontana KE. Suplementação com creatina associada ao treinamento resistido não altera as funções renal e hepática. Rev Bras Med Esporte. 2011;17(4):237-41.

3. Altimari LR, Okano AH, Trindade MCC, Cyrino ES, Tirapegui J. Efeito de oito semanas de suplementação com creatina monohidratada sobre o trabalho total relativo em esforços intermitentes máximos no cicloergômetro de homens treinados. Rev Bras Ciênc Farm. 2006;42(2):237-44.

4. Rawson ES, Volek JS. Effects of creatine supplementation and resistance training on muscle strength and weightlifting performance. J Strength Cond Res. 2003;17(4):822-31.

5. Willoughby DS, Rosene JM. Effects of oral creatine and resistance training on myogenic regulatory factor expression. Med Sci Sports Exerc. 2003;35(6):923-9.

6. Greenhaff PL. The nutritional biochemistry of creatine. J Nutr Biochem. 1997;8(11):610-8.

7. Gualano B, Roschel H, Lancha-Jr AH, Brightbill CE, Rawson ES. In sickness and in health: the widespread application of creatine supplementation. Amino Acids. 2012;43(2):519-29.

8. Aaberg E. Conceito e técnicas para o treinamento resistido. Barueri, SP: Manole; 2002.

9. Willardson JM, Burkett $L N$. The effect of rest interval length on bench press performance with heavy vs. light loads. J Strength Cond Res. 2006;20(2):396-9.

10. Polito MD, Farinatti PT. Considerações sobre a medida da pressão arterial em exercícios contra-resistência. Rev Bras Med Esporte. 2003;9(1):25-33.

11. Easton C, Turner S, Pitsiladis YP. Creatine and glycerol hyperhydration in trained subjects before exercise in the heat. Int I Sport Nutr Exerc Metab. 2007;17(1):70-91

12. Hile AM, Anderson JM, Fiala KA, Stevenson JH, Casa DJ, Maresh CM. Creatine supplementation and anterior compartment pressure during exercise in the heat in dehydrated men. J Athl Train. 2006;41(1):30-5.

13. Bemben MG, Bemben DA, Loftiss DD, Knehans AW. Creatine supplementation during resistance training in college football athletes. Med Sci Sports Exerc. 2001;33(10):1667-73.

14. Gualano B, Acquesta FM, Ugrinowitsch C, TricoliV, Serrão JC, Lancha Junior AH. Efeitos da suplementação de creatina sobre força e hipertrofia muscular: atualizaçōes. Rev Bras Med Esporte. 2010;16(3):219-223.

15. Olsen S, Aagaard P, Kadi F, Tufekovic G, Verney J, Olesen JL, et al. Creatine supplementation augments the increase in satellite cell and myonuclei number in human skeletal muscle induced by strength training. J Physiol. 2006;573(Pt 2):525-34.

16. Volek JS, Mazzetti SA, Farquhar WB, Barnes BR, Gómez AL, Kraemer WJ. Physiological responses to short-term exercise in the heat after creatine loading. Med Sci Sports Exerc. 2001;33(7):1101-8.

17. Spillane M, Schoch R, Cooke M, Harvey T, Greenwood M, Kreider R, et al. The effects of creatine ethyl ester supplementation combined with heavy resistance training on body composition, muscle performance, and serum and muscle creatine levels. J Int Soc Sports Nutr. 2009;6:6.

18. Kilduff LP, Georgiades E, James N, Minnion RH, Mitchell M, Kingsmore D, et al. The effects of creatine supplementation on cardiovascular, metabolic, and thermoregulatory responses during exercise in the heat in endurance-trained humans. Int J Sport Nutr Exerc Metab. 2004;14(4):443-60.

19. Beis LY, Polyviou T, Malkova D, Pitsiladis YP. The effects of creatine and glycerol hyperhydration on running economy in well trained endurance runners. J Int Soc Sports Nutr. 2011;8(1):24.

20. Deldicque L, Louis M, Theisen D, Nielens H, Dehoux M, Thissen JP, et al. Increased IGF mRNA in human skeletal muscle after creatine supplementation. Med Sci Sports Exerc. 2005;37(5):731-6.

21. Cooper R, Naclerio F, Allgrove J, Jimenez A. Creatine supplementation with specific view to exercise/ sports performance: an update. J Int Soc Sports Nutr. 2012;9(1):33.

22. Selberg O, Selberg D. Norms and correlates of bioimpedance phase angle in healthy human subjects, hospitalized patients, and patients with liver cirrhosis. Eur J Appl Physiol. 2002;86(6):509-16

23. Barbosa-Silva MC, Barros AJ, Wang J, Heymsfield SB, Pierson RN Jr. Bioelectrical impedance analysis: population reference values for phase angle by age and sex. Am J Clin Nutr. 2005;82(1):49-52 